\title{
Numerical Investigation of Supersonic Gas Jet Noise using Large Eddy Simulation
}

\author{
Lei Zhang ${ }^{a}$, Hao Wang and Wenjun Ruan \\ School of Energy and Power Engineering, Nanjing University of Science and Technology, Nanjing \\ 210094, China \\ a13770568711@163.com
}

Keywords: Jet flow, Acoustics, Large eddy simulation (LES), noise reduction.

\begin{abstract}
Noise of gas jet is one of the main noise sources for solid rocket motor, the jet flow field parameters plays an important role in the radiated noise generated. An implicit large eddy simulation (LES) methodology is performed of supersonic gas jet flows in this paper. The flow-induced noise generated by the jets is investigated by a computational acoustics methodology that couples the near field unsteady flow field data computed by LES with a surface integral acoustic method for noise prediction. Noise sources and integral surfaces are selected on the basis of turbulence characteristics. The calculation results show that the jet noise is strongly directional, with the angle of the measuring locations deviating from the jet central axis increases, the sound pressure level peak decreases, and it reaches maximum at the angle of $45^{\circ}$ away from the jet central axis. Comparisons of the jet mean flow, turbulent statistics, and jet noise results with experimental data of jets at similar flow conditions were done and reasonable agreement is observed. The results can be taken into consideration about reduction of solid rocket engine jet noise.
\end{abstract}

\section{Introduction}

The severe acoustic levels environment arising from supersonic turbulent exhaust jets of rocket engines induce severe random structural vibrations of vehicle components, encapsulated payloads, and ground support structures and equipment in the immediate vicinity of the launch pad. Additionally, these powerful impulse noise levels are of increasing concern to the researchers because of the hearing loss and other physiological impacts to personnel who work in close proximity to the launch pad. So the research on the gas jet noise is very important. Supersonic gas jets are one of the fascinating topics in fluid mechanics.

Mitchell and Lele[1] performed the DNS for both the flow and the sound radiated from subsonic and supersonic axisymmetric jets. The predicted sound was found to agree with predictions of Lighthill's acoustic analogy. McLaughlin et al. [2] has shown the jet noise generated by Mach waves is dominated by large scale turbulent structures for high Reynolds number supersonic jets. These large structures convert supersonically relative to the surrounding fluid based on the ambient speed of sound, and they contribute high noise levels near the downstream axial direction. Gao and Li [3] developed a computational acoustic tool for accurate prediction of edge tone noise, and improving our understanding of the noise generation mechanism. Bogey and Bailly [4] showed that inflow conditions, particularly the spatial structure of inflow disturbances, can significantly impact the development of jet flows and the radiated sound predicted by compressible LES at high Reynolds numbers $[5,6]$.

According to the generation mechanism and radiation characteristics of gas jet noise, based on the background of individual rocket launch, in this paper, the diffusion process of the gas jet is simulated by LES to obtain the distribution characteristic of parameters in jet field, and discussions are focused on variation law of the sound pressure level under different observation angles. 


\section{Theoretical model}

\subsection{Physical model}

High temperature and high pressure gas jet is a complicated turbulent flow process with chemical reaction. In order to make the numerical simulation of the process, the following simplified assumptions are made for the turbulent flow process of the gas jet:

The gas jet in the atmosphere is an unsteady free jet expansion process, and the solid particles in the gas jet are not considered.

The combustion gas is approximated as compressible and ideal gas jet.

The secondary influence factors such as the gravity of the combustion gases are ignored.

The chemical reaction of the gas and the atmosphere is not considered.

The LES model is used for the turbulent mixture between the gas and atmosphere.

Regardless of the solid wall around the acoustic reflex action.

\subsection{Mathematical model}

Flow control equation

After filtering for compressible flow continuity equation, momentum equation and energy equation [7]:

$$
\begin{aligned}
& \frac{\partial \bar{\rho}}{\partial t}+\frac{\partial\left(\bar{\rho} \tilde{u}_{i}\right)}{\partial x_{i}}=0 \\
& \frac{\partial\left(\bar{\rho} \tilde{u}_{i}\right)}{\partial t}+\frac{\partial\left(\bar{\rho} \tilde{u}_{j} \tilde{u}_{i}\right)}{\partial x_{j}}+\frac{\partial \bar{p}}{\partial x_{i}}=\frac{\partial\left(\bar{\sigma}_{i j}+\tau_{i j}\right)}{\partial x_{j}} \\
& \frac{\partial(\bar{\rho} \tilde{E})}{\partial t}+\frac{\partial\left[(\bar{\rho} \tilde{E}+\bar{p}) \tilde{u}_{i}\right]}{\partial x_{i}}=\frac{\partial}{\partial x_{i}}\left(q_{i}+C_{p} \frac{\mu}{\operatorname{Pr}} \frac{\partial \tilde{T}}{\partial x_{i}}+\tilde{u}_{j} \tau_{i j}+\tilde{u}_{j} \bar{\sigma}_{i j}\right)
\end{aligned}
$$

Where $\bar{\sigma}_{i j}$ and $\tau_{i j}$ are the Favre filtered viscous stress tensor and subgrid scale viscous stress tensor respectively. These are defined as

$$
\begin{aligned}
& \bar{\sigma}_{i j}=2 \mu \tilde{S}_{i j}-\frac{2}{3} \mu \tilde{S}_{m m} \delta_{i j} \\
& \tau_{i j}=\mu_{t}\left(2 \tilde{S}_{i j}-\frac{2}{3} \tilde{S}_{m m} \delta_{i j}\right)-\frac{2}{3} \bar{\rho} k^{S G S} \delta_{i j}
\end{aligned}
$$

Sound control equations

The Ffowcs Williams-Hawking (FW-H) equation is derived from the continuous and the momentum equation,

$$
\begin{aligned}
& \frac{1}{c_{0}^{2}} \frac{\partial^{2} p^{\prime}(x, t)}{\partial t^{2}}-\nabla^{2} p^{\prime}(x, t)=\frac{\partial^{2}}{\partial x_{i} \partial x_{j}}\left[T_{i j} H(f)\right] \\
& -\frac{\partial}{\partial x_{i}}\left[L_{i} \delta(f)\right]+\frac{\partial}{\partial t}\left[\left(\rho_{0} U_{i}\right) \delta(f)\right]
\end{aligned}
$$

Among that:

$$
\begin{aligned}
& U_{i}=\left[1-\left(\rho / \rho_{0}\right)\right] v_{i}+\rho u_{i} / \rho_{0}, \\
& L_{i}=P_{i j} n_{j}+\rho u_{i}\left(u_{n}-v_{n}\right)
\end{aligned}
$$

Where $p^{\prime}$ is far-field sound pressure, $P_{i j}$ is stress tensor, $H(f)$ is Heaviside function, $\delta(f)$ is Dirac delta function, $T_{i j}$ is Lighthill stress tensor given by,

$$
\begin{aligned}
& T_{i j}=\rho u_{i} u_{j}+P_{i j}-c_{0}^{2}\left(\rho-\rho_{0}\right) \delta_{i j} \\
& \text { Initial and boundary conditions }
\end{aligned}
$$


The parameter values of the air medium are the initial conditions of the computational area. The pressure boundary condition of inlet is determined by experiment that is $p=20 \mathrm{MPa}, T=3000 \mathrm{~K}$. The boundary of the nozzle and tail tube is a fixed wall. The exit of tail tube is connected with atmosphere, so the pressure boundary of outlet is the atmospheric parameter. The computation domain of numerical simulation, as be shown in Fig.1.

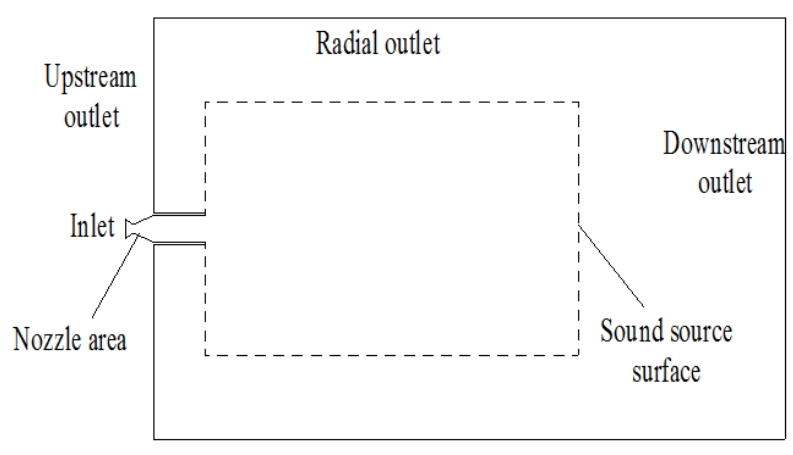

Fig. 1 Computational domain

\section{Numerical Results and Discussion}

\subsection{Flow field}

Fig. 2 shows the instantaneous flow field distribution features of gas jet. Figure 2(a) depicts contours of instantaneous velocity. Figure 2(b) depicts contours of instantaneous pressure along the jet axis. As you can see, the main flow characteristics of the intersection and reflection of expansion wave and compression wave, the boundary layer of gas jet. High pressure gas injection from the tail tube into the lower pressure atmosphere environment, on the one hand, the gas jet expansion makes it accelerate and produce pressure drop along the axial direction; on the other hand, the more expansion of the gas jet makes the pressure lower than ambient pressure, resulting in the reflection of the compression wave in the shear layer boundary of the gas jet. The interaction between the expansion wave, the reflected shock wave and the shear layer of the jet makes continuous alternation of the expansion wave and the compression wave in the supersonic less expansion gas jet. In the process, the structure of the expansion wave and compression wave is weakened because of the dissipation of the shock.

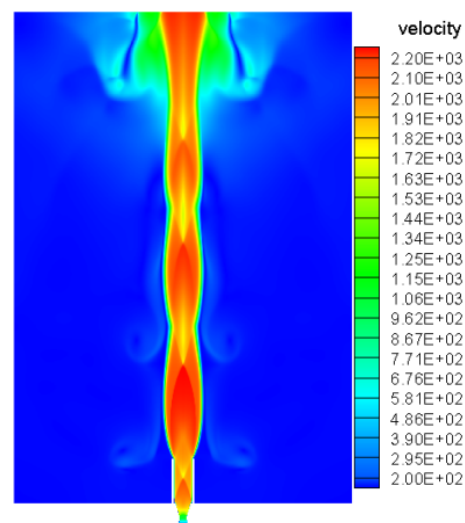

Fig. 2(a) Instantaneous velocity distribution

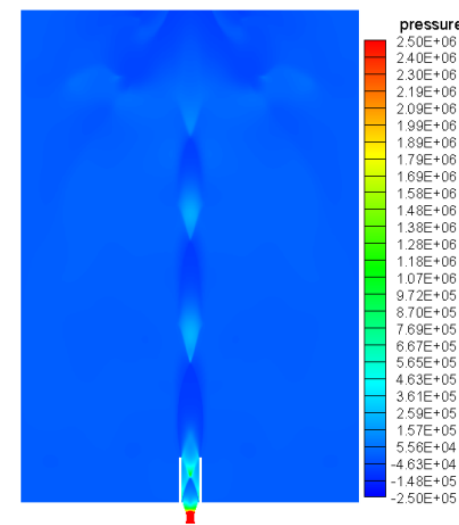

Fig. 2(b) Instantaneous pressure distribution

\subsection{Far-field acoustics}

Based on the flow field data, the FW-H surface integral acoustic method is used to predict the far-field jet noise at $\mathrm{R}=1.5 \mathrm{~m}$ from the nozzle exit center at polar angle $\theta$, defined as the angle between $+x$ and observer line R. By taking the Fourier transform on the time domain signal, the sound pressure level in the frequency domain is obtained.

Figure 3 shows the sound pressure level spectrum of the gas jet at the a few observer locations. As shown in Figure3, variations of sound pressure level spectrum at different observer locations have a certain similarity. With the increase of frequency, the jet noise sound pressure level first increases and 
then decreases in general, especially high frequency section above $6000 \mathrm{~Hz}$ down to smaller, and the sound pressure level is fluctuated within a certain range and the value is relatively small. The main components of jet noise performance in low frequency range, noise radiation energy is concentrated in less than $580 \mathrm{~Hz}$, and the peak sound pressure level will be significant around $300 \mathrm{~Hz}$. This is due to the low frequency band is mainly the result of large scale vortex movement, the sound power level is higher, while the high frequency section mainly performance for small scale vortex movement, the sound power level is lower. In the polar angle range $40^{\circ} \sim 116^{\circ}$, the sound pressure level within the band present a decreasing trend with the increase of polar angle of observation location.

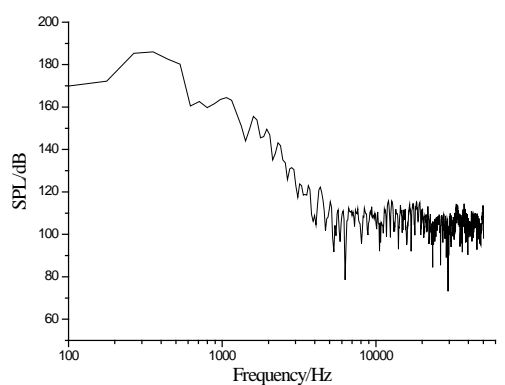

$\theta=450$

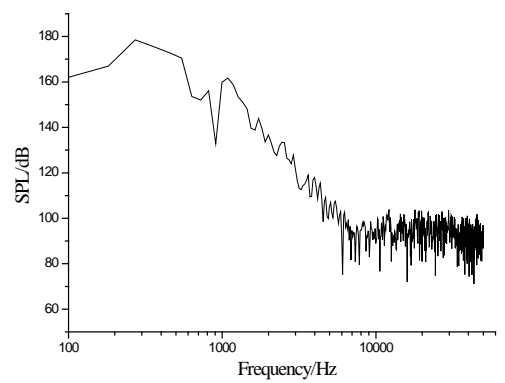

$\theta=750$

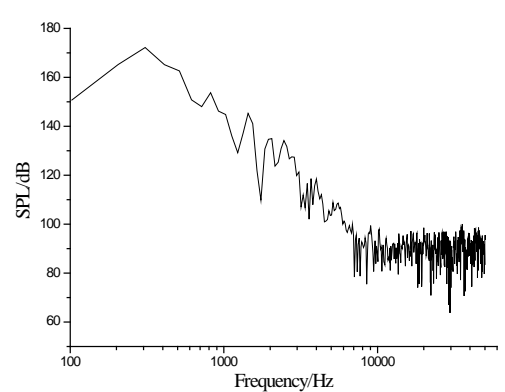

$\theta=1050$

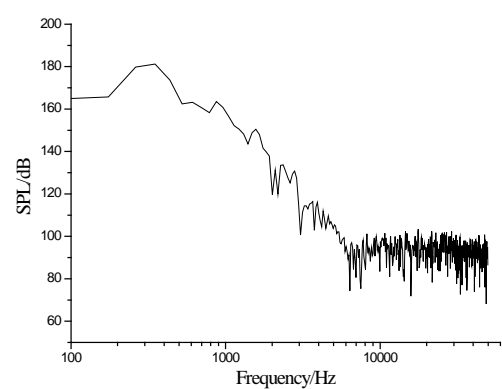

$\theta=600$

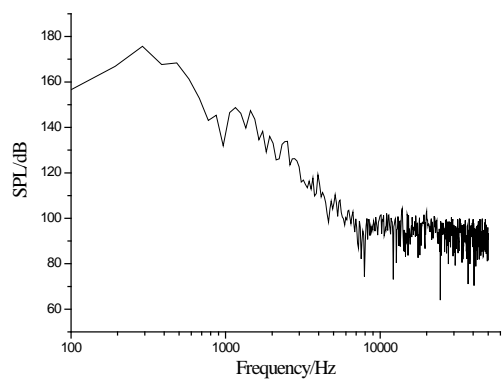

$\theta=900$

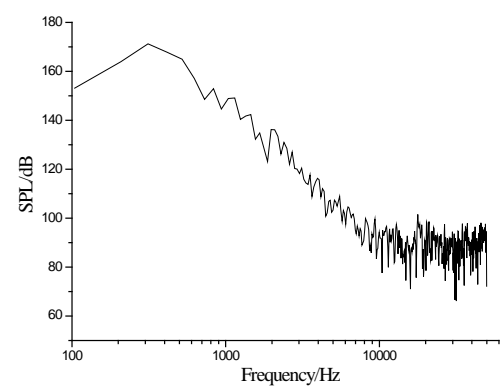

$\theta=1160$

Fig. 3 Spectrum of sound pressure level for different direction angles

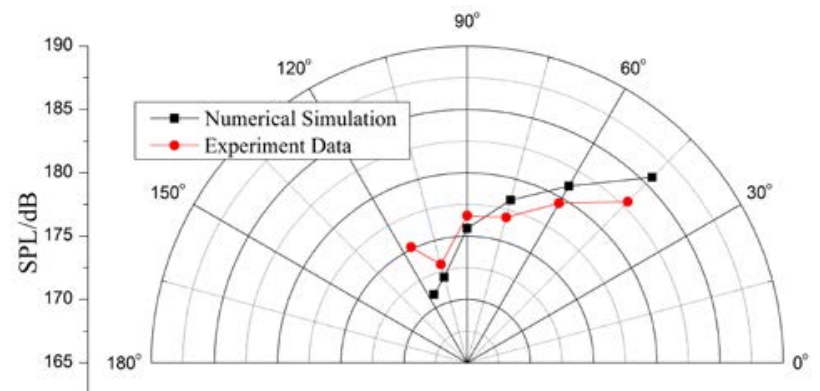

Fig. 4. Comparison of the predicted sound pressure level peak with experiment 
Figure 4 shows the variation trend of the sound pressure level (SPL) at different polar angles. It can be seen from Fig. 4 that the gas jet noise sound pressure level decreases with the increase of polar angle. At $\theta=60^{\circ}$, the sound pressure level is $185.7 \mathrm{~dB}$. When the angle is $116^{\circ}$, the sound pressure level is minimal, down to $171 \mathrm{~dB}$. The results show that the jet noise is not uniform in the circumferential direction, and the distribution law of the sound pressure level is the same as the experiment results showing the obvious directivity. As you can see, the calculated values are in good agreement with the experimental results. In the upstream direction of the maximum polar angle $116^{\circ}$, the difference between the numerical simulation and experiment is the larger, the difference is only $4.1 \mathrm{~dB}$, and only has an estimated maximum error of $2.4 \%$, which is mainly due to the calculation does not consider the wall reflects sound waves caused.

\section{Conclusions}

Base on the results of the numerical simulation, the following conclusions can be obtained:

(1) Flow and noise predictions have been made for a Laval nozzle at a hot supersonic flow condition. The instantaneous near field is examined by means of flow visualization, which demonstrates the main flow characteristics of the intersection and reflection of expansion wave and compression wave, the boundary layer of gas jet.

(2) For the far-field noise, variations of sound pressure level spectrum at different observer locations have a certain similarity. With the increase of frequency, the jet noise sound pressure level first increases and then decreases in general. The gas jet noise sound pressure level decreases with the increase of deviating from the direction angle. The jet noise is not uniform in the circumferential direction, and the distribution law of the sound pressure level is the same as the experiment results showing the obvious directivity.

\section{References}

[1]. Mitchell, B. E., Lele, S. K. and Moin P., Direct computation of the sound generated by vortex pairing in an axisymmetric jet. J. Fluid Mech., Vol. 383, 1999.

[2]. McLaughlin, D.K., Morrison, G. L., and Troutt, T. R., Reynolds number dependence on supersonic jet noise. AIAA Journal, Vol. 15, No.4, 1977.

[3]. Gao J.H., Li X. D., Large-Eddy simulation of the noise from a subsonic jet-edge system. AIAA 2010-4014, 2010.

[4]. Bogey, C. and Bailly, C., Computation of a high Reynolds number jet and its radiated noise using large eddy simulation base on explicit filtering. Computational Fluids, Vol. 33, 2006.

[5]. Rizzetta, D. P. and Visbal, M. R. , Large-Eddy simulation of supersonic cavity flow fields including flow control. AIAA, 2002-2853.

[6]. Aleksey M.S., Mikhail V.U., Applying Fluent software for jet noise generation modelling. AIAA, 2010-3843.

[7]. Andersson N., Eriksson L. E., and Davidson L., A study of Mach 0.75 jets and their radiated sound using large-eddy simulation. AIAA 2004-3024, 2004. 